\title{
Symplectic group methods and the Arthurs Kelly model of measurement in quantum mechanics
}

\author{
Arvind* \\ Department of Physical Sciences, Indian Institute of Science Education and Research (IISER) Mohali, \\ Sector 81 SAS Nagar, Manauli PO 140306, Punjab, India \\ S. Chaturved \\ Department of Physics, Indian Institute of Science Education and Research (IISER) Bhopal, \\ Bhopal Bypass Road, Bhauri, Bhopal 462066, India \\ N. Mukunda用 \\ Adjunct Professor, Department of Physics, Indian Institute of Science Education and Research (IISER) Bhopal, \\ Bhopal Bypass Road, Bhauri, Bhopal 462066, India
}

\begin{abstract}
We study the use of methods based on the real symplectic groups $S p(2 n, \mathcal{R})$ in the analysis of the Arthurs-Kelly model of proposed simultaneous measurements of position and momentum in quantum mechanics. Consistent with the fact that such measurements are in fact not possible, we show that the observable consequences of the Arthurs-Kelly interaction term are contained in the symplectic transformation law connecting the system plus apparatus variance matrices at an initial and a final time. The individual variance matrices are made up of averages and spreads or uncertainties for single hermitian observables one at a time, which are quantum mechanically well defined. The consequences of the multimode symplectic covariant Uncertainty Principle in the Arthurs-Kelly context are examined.
\end{abstract}

\section{INTRODUCTION}

The problem of understanding the measurement process in quantum mechanics has been of long standing interest, and has seen a significant revival in recent times [1]. The quantum Zeno effect [2], the concept of weak measurements [3, 4], and efforts to understand the Born Rule from more basic principles [5], may be mentioned in this context. Joint measurements of noncommuting observables, which is the main theme of this paper, have been considered by several authors [6 9]. Weak sequential measurements of non-commuting observables have also been considered for state tomography [10, 11].

Probably the earliest model of measurement in quantum mechanics is the one formulated by von Neumann in 1932 , very soon after the discovery of quantum mechanics itself [12]. In this model, both the system $\mathcal{S}$ being studied and the apparatus $\mathcal{A}$ are treated quantum mechanically, and the measurement is described by a suitable coupling term in the total Hamiltonian. The Born Rule remains as something to be imposed externally.

An interesting approach to the general measurement problem is due to Sudarshan from 1976 [13]. Here while the system $\mathcal{S}$ is of course quantum mechanical, the apparatus $\mathcal{A}$ is initially regarded as a classical system. However it is then recast as a quantum system subject to continuous super selection rules, and then the possibil-

\footnotetext{
* arvind@iiser.mohali.ac.in

$\dagger$ subhash@iiserb.ac.in

$\ddagger$ nmukunda@gmail.com
}

ity of coupling of $\mathcal{S}$ and $\mathcal{A}$ as parts of an overall quantum system is studied. In this approach, while the Born Rule remains 'unexplained', the well known restriction in quantum mechanics that only mutually commuting i. e., compatible - dynamical variables can be simultaneously measured, is derived.

As just mentioned, quantum mechanics does not permit simultaneous measurement of non-commuting dynamical variables such as a coordinate $\hat{q}$ and its canonically conjugate momentum $\hat{p}$. A very interesting approach to measurement in this situation, treating $\hat{q}$ and $\hat{p}$ on the same footing, is the Arthurs-Kelly $(A-K)$ proposal of 1964 [6]. In the simplest case where the system $\mathcal{S}$ is based on one canonical pair of hermitian Cartesian operators $\hat{q}$ and $\hat{p}$, the apparatus $\mathcal{A}$ is taken to be a quantum system involving two kinematically independent canonical pairs of operators $\hat{Q}_{1}, \hat{P}_{1}$ and $\hat{Q}_{2}, \hat{P}_{2}$. The idea is to use the commuting operators $\hat{Q}_{1}$ and $\hat{Q}_{2}$ to act as pointer positions to track the values of $\hat{q}$ and $\hat{p}$ respectively, using von Neumann type coupling terms in the total Hamiltonian.

An interesting consequence of the $A-K$ model, frequently mentioned as an important feature of it, is a kind of uncertainty principle for the pair $\hat{Q}_{1}$ and $\hat{Q}_{2}$, which a priori are compatible variables. It states that the lower bound on the product of their uncertainties is twice that for the familiar canonical $\hat{q}-\hat{p}$ pair, paying due attention to the differences in physical dimensions in the two cases, and this is ascribed to inherent and unavoidable extra noise in joint quantum measurements.

The purpose of the present work is to revisit the $A-K$ model, in particular to explore the use of methods based on the real symplectic groups in this context. As has 
been shown elsewhere, for quantum systems involving, say, $N$ canonical pairs of operators of the $\hat{q}-\hat{p}$ type, the most general statement of the multimode Uncertainty Principle is $S p(2 n, \mathcal{R})$ covariant, and is best understood and stated using the properties of these groups. As the original Arthurs-Kelly model involves a system with one degree of freedom and an apparatus with two degrees of freedom, the relevant group here is $S p(6, \mathcal{R})$.

The contents of the paper are arranged as follows. Section III recapitulates the kinematics and interaction term in the Arthurs-Kelly model, generalized to have two independent coupling constants. The solution of the Schrödinger equation for a general initial condition, as well as for product initial wavefunctions, are given. Section III identifies the Hamiltonian as a generator of $S p(6, \mathcal{R})$. This allows the solution to the operator Heisenberg equations of motion to be expressed via a matrix in $S p(6, \mathcal{R})$, as also the time dependent expectation values of the basic canonical variables. Section IV extends this approach to express the relation between the variance matrices at two different times as a symplectic congruence transformation. It is shown that all the observable consequences of the model are contained in such matrix relations. The consequences of the $S p(6, \mathcal{R})$ covariant statement of the Uncertainty Principle for general states of the combined system, as well as for special states of product form, are analyzed. The relevance of the Williamson normal forms of variance matrices is brought out. Section $[\mathrm{V}$ contains some concluding remarks.

\section{THE BASIC FEATURES OF THE $A$ - $K$ MODEL}

The kinematic structure of the $A-K$ model is given by three hermitian Cartesian position-momentum operator pairs: $\hat{q}$ and $\hat{p}$ for system $\mathcal{S}$; and $\hat{Q}_{1}, \hat{P}_{1}, \hat{Q}_{2}, \hat{P}_{2}$ for apparatus $\mathcal{A}$. We denote these operators collectively by $\hat{\xi}_{a}, a=1,2, \ldots, 6$ :

$$
\hat{\xi}_{1}=\hat{q}, \hat{\xi}_{2}=\hat{p}, \hat{\xi}_{3}=\hat{Q}_{1}, \hat{\xi}_{4}=\hat{P}_{1}, \hat{\xi}_{5}=\hat{Q}_{2}, \hat{\xi}_{6}=\hat{P}_{2} .
$$

The canonical commutation relations (CCR) are:

$$
\left[\hat{\xi}_{a}, \hat{\xi}_{b}\right]=i \hbar \beta_{a b}, \quad \beta=\left(\begin{array}{ccc}
i \sigma_{2} & 0 & 0 \\
0 & i \sigma_{2} & 0 \\
0 & 0 & i \sigma_{2}
\end{array}\right) .
$$

The natural covariance group of these CCR's is the noncompact real symplectic group $S p(6, \mathcal{R})$. This will be defined and exploited in succeeding Sections. More details about this group and $S p(2 n, \mathcal{R})$ can be found in [14].

The measurement is described using the $A$ - $K$ coupling of $\mathcal{S}$ and $\mathcal{A}$ with the interaction Hamiltonian

$$
\hat{H}=K_{1} \hat{q} \hat{P}_{1}+K_{2} \hat{p} \hat{P}_{2} .
$$

Which can be considered as the total Hamiltonian if we neglect the 'free' Hamiltonians for $\mathcal{S}$ and for $\mathcal{A}$ separately.
Here we allow independent choices of the real coupling constants $K_{1}$ and $K_{2}$ in general which are of course of different physical dimensions. This structure for $\hat{H}$ corresponds to $\hat{Q}_{1}$ being the pointer position for measuring $\hat{q}$, and $\hat{Q}_{2}$ for measuring $\hat{p}$. Quantum mechanics permits simultaneous measurements of $\hat{Q}_{1}$ and $\hat{Q}_{2}$, but not of $\hat{q}$ and $\hat{p}$. The aim is to learn as much as quantum mechanics allows about the latter from the former, based on the measurement interaction (3).

A general pure state Schrödinger wave function for $\mathcal{S} \oplus$ $\mathcal{A}$ is written as $\Psi\left(q ; Q_{1}, Q_{2}\right)$ with squared norm

$$
\|\Psi\|^{2}=\int_{-\infty}^{\infty} d q \int_{-\infty}^{\infty} d Q_{1} \int_{-\infty}^{\infty} d Q_{2}\left|\Psi\left(q ; Q_{1}, Q_{2}\right)\right|^{2} .
$$

The solution of the time dependent Schrödinger equation

$$
i \hbar \frac{\partial}{\partial t} \Psi\left(q ; Q_{1}, Q_{2} ; t\right)=\hat{H} \Psi\left(q ; Q_{1}, Q_{2} ; t\right)
$$

is easily obtained, using for instance the fact that $\hat{P}_{1}$ and $\hat{P}_{2}$ are both constants of motion. The result is

$$
\begin{gathered}
\Psi\left(q ; Q_{1}, Q_{2} ; t\right)=\frac{1}{2 \pi \hbar} \int_{-\infty}^{\infty} \int_{-\infty}^{\infty} d P_{2} d Q_{2}^{\prime} \mathrm{e}^{\frac{i}{\hbar}\left(Q_{2}-Q_{2}^{\prime}\right) P_{2}} \\
\times \Psi\left(q-K_{2} t P_{2} ; Q_{1}-K_{1} t\left(q-K_{2} \frac{t}{2} P_{2}\right), Q_{2}^{\prime} ; 0\right) .
\end{gathered}
$$

At any time $t>0$, a joint measurement of $\hat{Q}_{1}$ and $\hat{Q}_{2}$ (permitted by quantum mechanics) yields results $Q_{1}, Q_{2}$ with the joint probability distribution $P\left(Q_{1}, Q_{2} ; t\right)$ determined by the Born Rule:

$$
P\left(Q_{1}, Q_{2} ; t\right)=\int_{-\infty}^{\infty} d q\left|\Psi\left(q ; Q_{1}, Q_{2} ; t\right)\right|^{2} .
$$

For a general initial $\Psi\left(q ; Q_{1}, Q_{2} ; 0\right)$ this expression cannot be simplified in any significant manner. In case however the initial wave function for $\mathcal{S} \oplus \mathcal{A}$ is the product of a general pure state wave function $\psi$ for $\mathcal{S}$ and two independent real centered Gaussians for $\mathcal{A}$ :

$$
\begin{gathered}
\Psi\left(q ; Q_{1}, Q_{2} ; 0\right)=\sqrt{\frac{2}{\pi}} \frac{1}{\left(b_{1} b_{2}\right)^{1 / 4}} \psi(q) e^{-Q_{1}^{2} / b_{1}-Q_{2}^{2} / b_{2}}, \\
b_{1}, b_{2}>0, \quad \int_{-\infty}^{\infty} d q|\psi(q)|^{2}=1,
\end{gathered}
$$

Eq. (6) simplifies to

$$
\begin{aligned}
& \Psi\left(q ; Q_{1}, Q_{2} ; t\right)=\frac{1}{\sqrt{2}} \frac{1}{\pi \hbar}\left(\frac{b_{2}}{b_{1}}\right)^{1 / 4} \\
& \quad \times \int_{-\infty}^{\infty} d P_{2} e^{i Q_{2} P_{2} / \hbar} \psi\left(q-K_{2} t P_{2}\right) \\
& \quad \times e^{-\left(Q_{1}-K_{1} t q+K_{1} K_{2} \frac{t^{2}}{2} P_{2}\right)^{2} / b_{1}-b_{2} P_{2}^{2} / 4 \hbar^{2}} .
\end{aligned}
$$

The probability distribution $P\left(Q_{1}, Q_{2} ; t\right)$ also simplifies to some extent and, after some algebra, takes the form:

$$
P\left(Q_{1}, Q_{2} ; t\right)=\frac{1}{2} \frac{1}{\left(\pi \hbar K_{2} t\right)^{2}} \sqrt{\frac{b_{2}}{b_{1}}} \int_{-\infty}^{\infty} d q e^{-\left(Q_{1}-K_{1} t q\right)^{2} / b_{1}} \times
$$




$$
\begin{gathered}
\left|\int_{-\infty}^{\infty} d q^{\prime} e^{-i Q_{2} q^{\prime} / \hbar K_{2} t} \psi\left(q^{\prime}\right) e^{-X(t)\left(q-q^{\prime}\right)^{2}-\left(Q_{1}-K_{1} t q^{\prime}\right)^{2} / 2 b_{1}}\right|^{2}, \\
\text { with } X(t)=\frac{b_{2}}{\left(2 \hbar K_{2} t\right)^{2}}-\frac{K_{1}^{2} t^{2}}{4 b_{1}} .
\end{gathered}
$$

In the original $A-K$ model, and in some later work as well, some more simplifying assumptions were made. In suitable units, including $\hbar=1$, (i) $K_{1}=K_{2}=K$; (ii) $b_{1}=b_{2}^{-1}=b$; (iii) $K t=1$. In this situation, Eq. (10) simplifies one more step, since $X(t)=0$ and the $q$ integration can be carried out:

$P\left(Q_{1}, Q_{2} ; t\right)=\frac{1}{\sqrt{2 \pi^{2} b}}\left|\int_{-\infty}^{\infty} d q^{\prime} \psi\left(q^{\prime}\right) e^{-i q^{\prime} Q_{2}-\left(q^{\prime}-Q_{1}\right)^{2} / 2 b}\right|^{2}$

We will however work with the more general expression (10), especially since the simplifying assumptions mentioned conflict with $K_{1}$ and $K_{2}$ being of different physical dimensions, while $b_{1}$ and $b_{2}$ are both squared lengths.

\section{THE GROUP $S p(6, \mathcal{R})$ AND ITS USES}

The CCR's (2) are preserved by real linear canonical transformations acting on $\hat{\xi}_{a}$. These form the 21parameter noncompact real symplectic group in 6 real dimensions defined by

$S p(6, \mathcal{R})=\left\{S=\left(S_{a b}\right)=\operatorname{real} 6 \times 6\right.$ matrix $\left.\mid S^{T} \beta S=\beta\right\}$,

where $\beta$ is the symplectic metric matrix given in Eq. (2). Thus we have:

$$
\begin{aligned}
& S \in S p(6, \mathcal{R}), \hat{\xi}_{a}^{\prime}=S_{b a} \hat{\xi}_{b} \Rightarrow\left[\hat{\xi}_{a}^{\prime}, \hat{\xi}_{b}^{\prime}\right]=i \hbar \beta_{a b}, \\
& \hat{\xi}_{a}^{\prime}=\mathcal{U}(S) \hat{\xi}_{a} \mathcal{U}(S)^{-1}
\end{aligned}
$$

where the unitary operators $\mathcal{U}(S)$ are determined up to signs by $S$ and obey

$$
\mathcal{U}\left(S^{\prime}\right) \mathcal{U}(S)= \pm \mathcal{U}\left(S^{\prime} S\right)
$$

thus constituting the two-valued metaplectic unitary representation of $S p(6, \mathcal{R})$ [14, 15]. Since $\hat{\xi}_{1,3,5}$ are lengths and $\hat{\xi}_{2,4,6}$ are momenta, each element $S_{b a}$ of $S$ carries a suitable physical dimension. The $\mathcal{U}(S)$ are (products of) exponentials of antihermitian quadratic expressions in the $\hat{\xi}_{a}$.

The relevance of $S p(6, \mathcal{R})$ arises from the fact that the Hamiltonian (3) is quadratic in the $\hat{\xi}$ 's. We have

$$
\begin{gathered}
\hat{H}=\frac{1}{2} h_{a b} \hat{\xi}_{a} \hat{\xi}_{b}, \quad h_{a b}=h_{b a}, \\
h=\left(\begin{array}{cccccc}
0 & 0 & 0 & K_{1} & 0 & 0 \\
0 & 0 & 0 & 0 & 0 & K_{2} \\
0 & 0 & 0 & 0 & 0 & 0 \\
K_{1} & 0 & 0 & 0 & 0 & 0 \\
0 & 0 & 0 & 0 & 0 & 0 \\
0 & K_{2} & 0 & 0 & 0 & 0
\end{array}\right),
\end{gathered}
$$

the only nonzero elements of $h$ being $h_{14}=h_{41}=K_{1}$ and $h_{26}=h_{62}=K_{2}$. Therefore we get

$$
\begin{gathered}
{\left[\hat{H}, \hat{\xi}_{a}\right]=i \hbar J_{b a} \hat{\xi}_{b}, \quad\left[\hat{H},\left[\hat{H}, \hat{\xi}_{a}\right]\right]=-\hbar^{2}\left(J^{2}\right)_{b a} \hat{\xi}_{b}, \quad \ldots} \\
J=h \beta=\left(\begin{array}{cccccc}
0 & 0 & -K_{1} & 0 & 0 & 0 \\
0 & 0 & 0 & 0 & -K_{2} & 0 \\
0 & 0 & 0 & 0 & 0 & 0 \\
0 & K_{1} & 0 & 0 & 0 & 0 \\
0 & 0 & 0 & 0 & 0 & 0 \\
-K_{2} & 0 & 0 & 0 & 0 & 0
\end{array}\right) \\
J^{2}=K_{1} K_{2}\left(\begin{array}{cccccc}
0 & 0 & 0 & 0 & 0 & 0 \\
0 & 0 & 0 & 0 & 0 & 0 \\
0 & 0 & 0 & 0 & 0 & 0 \\
0 & 0 & 0 & 0 & -1 & 0 \\
0 & 0 & 0 & 0 & 0 & 0 \\
0 & 0 & 1 & 0 & 0 & 0
\end{array}\right), \quad J^{3}=0 .
\end{gathered}
$$

This real matrix $J$ obeys

$$
J^{T} \beta+\beta J=0, \quad \text { i.e. }(\beta J)^{J}=\beta J .
$$

As a result, the Heisenberg equations of motion for $\hat{\xi}_{a}(t)$ are explicitly solvable, containing only three terms:

$$
\begin{aligned}
\hat{\xi}_{a}(t) & =e^{i \hat{H} t / \hbar} \hat{\xi}_{a} e^{-i \hat{H} t / \hbar} \\
& =\hat{\xi}_{a}-t J_{b a} \hat{\xi}_{b}+\frac{t^{2}}{2}\left(J^{2}\right)_{b a} \hat{\xi}_{b} \\
& =\left(S(t)^{T} \hat{\xi}\right)_{a}, \\
S(t) & =e^{-t J}=\left(\begin{array}{cccccc}
1 & 0 & t K_{1} & 0 & 0 & 0 \\
0 & 1 & 0 & 0 & t K_{2} & 0 \\
0 & 0 & 1 & 0 & 0 & 0 \\
0 & -t K_{1} & 0 & 1 & -\frac{t^{2}}{2} K_{1} K_{2} & 0 \\
0 & 0 & 0 & 0 & 1 & 0 \\
t K_{2} & 0 & \frac{t^{2}}{2} K_{1} K_{2} & 0 & 0 & 1
\end{array}\right) \\
& \in S p(6, \mathcal{R})
\end{aligned}
$$

For any solution $|\Psi(t)\rangle$ of the time dependent Schrödinger equation (5), the symplectic matrix $S(t)$ determines the evolution of the expectation values of the operators $\hat{\xi}_{a}$ :

$$
\begin{aligned}
\xi_{a}(t) & =\left\langle\Psi(t)\left|\hat{\xi}_{a}\right| \Psi(t)\right\rangle \\
& =\left\langle\Psi(0)\left|\hat{\xi}_{a}(t)\right| \Psi(0)\right\rangle \\
& =S(t)_{b a} \xi_{b}(0) .
\end{aligned}
$$

For $a=3$ and 5 we get:

$$
\begin{aligned}
& Q_{1}(t)=S(t)_{b 3} \xi_{b}(0)=Q_{1}(0)+t K_{1} q(0)+\frac{t^{2}}{2} K_{1} K_{2} P_{2}(0), \\
& Q_{2}(t)=S(t)_{b 5} \xi_{b}(0)=Q_{2}(0)+t K_{2} p(0)-\frac{t^{2}}{2} K_{1} K_{2} P_{1}(0) .
\end{aligned}
$$

Here, $q(0)$ and $p(0)$ are the quantum mechanical expectation values of $\hat{q}$ and $\hat{p}$ of $\mathcal{S}$ in the state $|\Psi(0)\rangle$, obtained from independent measurements of these operators one at a time. The relations between the expectation values $Q_{1}(t), Q_{2}(t)$ of $\hat{Q}_{1}, \hat{Q}_{2}$ in $|\Psi(t)\rangle$ and $q(0), p(0)$ 
are encoded in the matrix $S(t)$. The 'pointer readings' $Q_{1}(t), Q_{2}(t)$ reveal the properties $q(0), p(0)$ of $\mathcal{S}$ consistent with $\left[\hat{Q}_{1}, \hat{Q}_{2}\right]=0,[\hat{q}, \hat{p}] \neq 0$.

In case $|\Psi(0)\rangle$ is of the product form (8), Eqs. (20) simplify to

$$
Q_{1}(t)=t K_{1} q(0), \quad Q_{2}(t)=t K_{2} p(0) .
$$

\section{SYMPLECTIC TRANSFORMATION LAW FOR VARIANCE MATRICES}

We first recall the $S p(6, \mathcal{R})$ covariant form of the Uncertainty Principle for general states of the composite system $\mathcal{S} \oplus \mathcal{A}$. The state is in general a mixed one described by a density matrix $\hat{\rho}$; in the pure case $\hat{\rho}=|\Psi\rangle\langle\Psi|$. The 6 dimensional real symmetric positive definite variance matrix $V=\left(V_{a b}\right)$ is defined by

$$
\begin{aligned}
V_{a b} & =\operatorname{Tr}\left[\hat{\rho} \frac{1}{2}\left\{\hat{\xi}_{a}, \hat{\xi}_{b}\right\}\right]-\left\langle\hat{\xi}_{a}\right\rangle\left\langle\hat{\xi}_{b}\right\rangle, \\
\left\langle\hat{\xi}_{a}\right\rangle & =\operatorname{Tr}\left(\hat{\rho} \hat{\xi}_{a}\right) .
\end{aligned}
$$

Under $S p(6, \mathcal{R})$ action on $\hat{\rho}$, the effect on $V$ is a matrix congruence transformation:

$$
S \in S p(6, \mathcal{R}): \hat{\rho}^{\prime}=\mathcal{U}(S)^{-1} \hat{\rho} \mathcal{U}(S) \Rightarrow V^{\prime}=S^{T} V S .
$$

The statement of the Uncertainty Principle in $S p(6, \mathcal{R})$ covariant form is

$$
V+\frac{i}{2} \hbar \beta \geq 0
$$

Every matrix $V$ obeying this condition is realizable (in general in infinitely many ways) as the variance matrix of some physical state. In particular, if $V$ is physically realizable, then so is $S^{T} V S$ for any $S \in S p(6, \mathcal{R})$ [16].

Ignoring for the moment the physical dimensions of each $V_{a b}$ and each $S_{a b}$, a given numerical matrix $V$ which is real symmetric positive definite and obeys $V+\frac{i}{2} \beta \geq 0$ can be written in the form

$$
\begin{aligned}
& V=S_{0}^{T} V_{d} S_{0}, \quad S_{0} \in S p(6, \mathcal{R}), \\
& V_{d}=\operatorname{diag}\left(\kappa_{1}, \kappa_{1}, \kappa_{2}, \kappa_{2}, \kappa_{3}, \kappa_{3}\right), \\
& \kappa_{j} \geq \frac{1}{2}, \quad j=1,2,3 .
\end{aligned}
$$

This is the Williamson normal form of $V$ and here $S_{0}$ too is purely numerical. For our problem with each $V_{a b}, S_{a b}$ carrying definite dimensions, we need a modified normal form which is:

$$
\begin{aligned}
V= & S_{0}^{\prime} T V_{d}^{\prime} S_{0}^{\prime}, \quad S_{0}^{\prime} \in S p(6, \mathcal{R}), \\
V_{d}^{\prime}= & \operatorname{diag}\left(\kappa_{1}, \kappa_{1}^{\prime}, \kappa_{2}, \kappa_{2}^{\prime}, \kappa_{3}, \kappa_{3}^{\prime}\right), \quad \kappa_{j}, \kappa_{j}^{\prime}>0, \\
& \kappa_{j} \kappa_{j}^{\prime} \geq \frac{\hbar^{2}}{4}, \quad j=1,2,3 .
\end{aligned}
$$

Now each $\kappa_{j}$ is a squared length and each $\kappa_{j}^{\prime}$ a squared momentum. The passage from (25) to (26) involves reciprocal scale transformations within each canonical pair, and these are elements of $S p(6, \mathcal{R})$.
Now we apply these results to the $A-K$ model. For simplicity we limit ourselves to pure states $|\Psi(0)\rangle$ at $t=$ 0 , though this is not essential. Then the variance matrix $V(t)$ evolves via $S(t) \in S p(6, \mathcal{R})$ in Eq. (18):

$$
\begin{aligned}
V_{a b}(t)= & \left\langle\Psi(t)\left|\frac{1}{2}\left\{\hat{\xi}_{a}, \hat{\xi}_{b}\right\}\right| \Psi(t)\right\rangle \\
& -\left\langle\Psi(t)\left|\hat{\xi}_{a}\right| \Psi(t)\right\rangle\left\langle\Psi(t)\left|\hat{\xi}_{b}\right| \Psi(t)\right\rangle . \\
V(t)= & S(t)^{T} V(0) S(t),
\end{aligned}
$$

Let us focus on the uncertainties or spreads in the pointer positions, $\left(\Delta Q_{1}\right)^{2}=V_{33}$ and $\left(\Delta Q_{2}\right)^{2}=V_{55}$. In the diagonal form of $V_{d}^{\prime}$ in Eq. (26), we have $\Delta Q_{1}=\kappa_{2}^{1 / 2}, \Delta Q_{2}=$ $\kappa_{3}^{1 / 2}$ and there is no lower bound on $\kappa_{2} \kappa_{3}$. Based on the general $S p(6, \mathcal{R})$ transformation rule (23) for $V$, it is easy to see that for any chosen $t>0$, we can always choose $V(0)$ so that $\left(\Delta Q_{1}\right)(t)$ and $\left(\Delta Q_{2}\right)(t)$ are each as small and positive as we wish. Thus as expected, quantum mechanics does not imply any universal lower bound for the uncertainty product $\Delta Q_{1} \Delta Q_{2}$.

To study the time dependences of $\left(\Delta Q_{1}\right)(t)$ and $\left(\Delta Q_{2}\right)(t)$ further, we need to evaluate $V_{33}(t), V_{55}(t)$ respectively. In each case, since many elements of $S(t)$ vanish, only six terms remain, three of direct type and three cross terms:

$$
\begin{aligned}
& \left(\Delta Q_{1}\right)^{2}(t)=V_{33}(t)=S_{a 3}(t) S_{b 3}(t) V_{a b}(0) \\
& =V_{33}(0)+\left(t K_{1}\right)^{2} V_{11}(0)+\frac{1}{4}\left(t^{2} K_{1} K_{2}\right)^{2} V_{66}(0) \\
& +2 t K_{1} V_{13}(0)+t^{2} K_{1} K_{2} V_{36}(0)+t^{3} K_{1}^{2} K_{2} V_{16}(0) \\
& =\left(\Delta Q_{1}\right)^{2}(0)+\left(t K_{1}\right)^{2}(\Delta q)^{2}(0)+\frac{1}{4}\left(t^{2} K_{1} K_{2}\right)^{2}\left(\Delta P_{2}\right)^{2}(0)
\end{aligned}
$$$$
+3 \text { cross terms; }
$$

$$
\begin{aligned}
& \left(\Delta Q_{2}\right)^{2}(t)=V_{55}(t)=S_{a 5}(t) S_{b 5}(t) V_{a b}(0) \\
& =\left(\Delta Q_{2}\right)^{2}(0)+\left(t K_{2}\right)^{2}(\Delta p)^{2}(0)+\frac{1}{4}\left(t^{2} K_{1} K_{2}\right)^{2}\left(\Delta P_{1}\right)^{2}(0)
\end{aligned}
$$

+3 cross terms.

In comparison with Eq. (21), we see that just as the mean values $Q_{1}(t), Q_{2}(t)$ lead to the mean values $q(0), p(0)$ of the operators $\hat{q}, \hat{p}$ of $\mathcal{S}$, now $\left(\Delta Q_{1}\right)(t),\left(\Delta Q_{2}\right)(t)$ yield the spreads $(\Delta q)(0),(\Delta p)(0)$ in $\hat{q}, \hat{p}$ at $t=0$.

Let us finally consider $|\Psi(0)\rangle$ to be of the product form Eq.(8). The initial variance matrix $V(0)$ is now block diagonal:

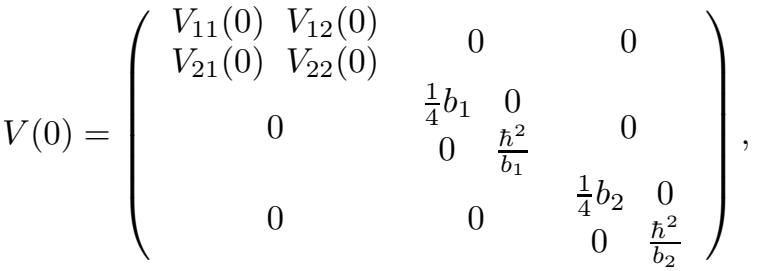

$$
\begin{aligned}
& V_{11}(0)=(\Delta q)^{2}(0) \text {, } \\
& V_{22}(0)=(\Delta p)^{2}(0) \text {, } \\
& V_{12}(0)=(\Delta q \Delta p)(0) \text {. }
\end{aligned}
$$


All cross terms in Eqns. (28) \& (29) vanish, and we find:

$$
\begin{aligned}
& \left(\Delta Q_{1}\right)^{2}(t)=\left(t K_{1}\right)^{2}(\Delta q)^{2}(0)+\frac{1}{4 b_{2}}\left(b_{1} b_{2}+\left(t^{2} \hbar K_{1} K_{2}\right)^{2}\right), \\
& \left(\Delta Q_{2}\right)^{2}(t)=\left(t K_{2}\right)^{2}(\Delta p)^{2}(0)+\frac{1}{4 b_{1}}\left(b_{1} b_{2}+\left(t^{2} \hbar K_{1} K_{2}\right)^{2}\right) .
\end{aligned}
$$

Using $\Delta q(0) \Delta p(0) \geq \hbar / 2$, we find a lower bound for $\Delta Q_{1}(t) \Delta Q_{2}(t)$ :

$$
\begin{aligned}
& \left(\Delta Q_{1}(t) \Delta Q_{2}(t)\right)^{2}=\left(t^{2} K_{1} K_{2}\right)^{2}(\Delta q(0) \Delta p(0))^{2} \\
& +\frac{1}{4}\left(b_{1} b_{2}+\left(t^{2} \hbar K_{1} K_{2}\right)^{2}\right) \\
& \quad \times\left(\frac{\left(t K_{1}\right)^{2}}{b_{1}}(\Delta q)^{2}(0)+\frac{\left(t K_{2}\right)^{2}}{b_{2}}(\Delta p)^{2}(0)\right) \\
& +\frac{1}{16 b_{1} b_{2}}\left(b_{1} b_{2}+\left(t^{2} \hbar K_{1} K_{2}\right)^{2}\right)^{2} \\
& \geq\left(b_{1} b_{2}+2 \sqrt{b_{1} b_{2}} \hbar t^{2} K_{1} K_{2}+\left(t^{2} \hbar K_{1} K_{2}\right)^{2}\right)^{2} / 16 b_{1} b_{2},
\end{aligned}
$$

i.e.,

$$
\Delta Q_{1}(t) \Delta Q_{2}(t) \geq \frac{1}{4 \sqrt{b_{1} b_{2}}}\left(\sqrt{b_{1} b_{2}}+t^{2} \hbar K_{1} K_{2}\right)^{2} .
$$

However, as is to be expected, this 'Uncertainty Principle' for $\Delta Q_{1}(t) \Delta Q_{2}(t)$ depends both on the parameters $K_{1}, K_{2}$ in the Hamiltonian (3) and on the parameters $b_{1}, b_{2}$ in the initial product wave function (8).

\section{CONCLUDING REMARKS}

In this paper we have shown the usefulness of methods based on the symplectic group $S p(6, \mathcal{R})$ for analyzing the consequences of the Arthurs-Kelly model of measurement in quantum mechanics. All physically meaningful results are expressed in the connections between time dependent variance matrices at different times. These connections are stated in terms of specific real six dimensional symplectic matrices. The variance matrices are made up of averages and spreads of one observable at a time. So even though the model is an attempt to give meaning to simultaneous measurements of position and momentum in quantum mechanics, only the variance matrices are involved in the statement of observable consequences of the model. The presence of two independent coupling constants in the interaction Hamiltonian allows us to study various options of measurement sequences with some flexibility.

From the Eqns. (21) \& (32) it is clear that, a measurement of commuting variables $\hat{Q}_{1}$ and $\hat{Q}_{2}$ at time $t$, reveals information about non-commuting variable $\hat{q}$ and $\hat{p}$ at $t=0$. If these measurements are repeated several times, the mean values and the variances at time $t=0$ of $\hat{q}$ and $\hat{p}$ can be estimated. These estimates will contain additional noise because we are estimating non-commuting observables. These additional noise terms are the last terms in Eqns. (32). The strength of the $\hat{q}$ measurement is determined by $t K_{1}$ and $b_{1}$. The larger the values of $t K_{1}$ the stronger is the measurement and the smaller the value of $b_{1}$, the stronger is the measurement. In a similar manner, the strength of the $\hat{p}$ measurement is dictated by the values of the parameters $t K_{2}$ and $b_{2}$.

The formalism presented here allows us to explore different regimes, for instance, we can easily switch off one measurement by setting $K_{1}$ or $K_{2}$ to zero. We can perform sequential measurements by first having a non-zero $K_{1}$ with $K_{2}$ set to zero and vice versa. In such a sequential measurement, the first measurement is required to be weak, in the sense that $t K_{1}$ is small or $b_{1}$ is large. The second measurement could be a strong one and close to a projective measurement.

We can try to estimate the state of the system from the measurements of $\hat{Q}_{1}$ and $\hat{Q}_{2}$. Such estimates can be done in many ways: independent measurements, sequential measurements, and joint measurements. What we would like to emphasize is that symplectic techniques are useful in all these scenarios. Some of these aspects will be taken up elsewhere.

\section{ACKNOWLEDGMENTS}

One of the authors (NM) thanks the Indian National Science Academy for award of INSA Distinguished Professorship during whose tenure this work was initiated.
[1] E. P. Wigner, American Journal of Physics 31, 6 (1963).

[2] B. Misra and E. C. G. Sudarshan, J. Mathematical Phys. 18, 756 (1977).

[3] Y. Aharonov, D. Z. Albert, and L. Vaidman, Phys. Rev. Lett. 60, 1351 (1988)

[4] N. D. H. Dass, Current Science 109, 1966 (2015).

[5] A. Patel and P. Kumar, Phys. Rev. A 96, 022108 (2017)

[6] E. Arthurs and J. L. Kelly, The Bell System Technical Journal 44, 725 (1965).

[7] S. Roy, Physics Letters A 377, 2011 (2013)
[8] P. Busch and P. J. Lahti, Phys. Rev. D 29, 1634 (1984)

[9] D. M. Appleby, Journal of Physics A: Mathematical and General 31,

[10] D. Das and Arvind, Phys. Rev. A 89, 062121 (2014)

[11] D. Das and Arvind, Journal of Physics A: Mathematical and Theoret

[12] J. von Neumann, Mathematical Foundations of Quantum Mechanics (Princeton University Press, Princeton NJ, 1955).

[13] E. C. G. Sudarshan, Pramana 6, 117 (1976)

[14] Arvind, B. Dutta, N. Mukunda, and R. Simon, Pramana 45, 471 (1995). 
[15] Arvind, B. Dutta, C. L. Mehta, and N. Mukunda, Phys. Rev. A 50, 39 (1994).
[16] R. Simon, E. C. G. Sudarshan, and N. Mukunda, Phys. Rev. A 37, 3028 (1988). 